\title{
A Novel Telerobotic System to Remotely Navigate Standard Electrophysiology Catheters
}

\author{
E Marcelli, L Cercenelli, G Plicchi \\ Università di Bologna, Bologna, Italy
}

\begin{abstract}
Remote manipulation of electrophysiology (EP) catheters by means of magnetically-guided or robotically controlled navigation systems has been recently proposed; however, these systems usually require dedicated catheters and devices. This study aims at evaluating the feasibility of using a novel Telerobotic System (TS) to remotely manipulate standard steerable EP catheters. In order to prevent cardiac tissue damage the TS was equipped with a force sensor to measure the resistance encountered by the catheter while advancing. The use of this novel TS was evaluated in three sheep, by performing remote navigation of a standard EP catheter to selected catheter-endocardium contact targeted sites in the right atrium. Remote catheter navigation by means of the TS was achieved for all targets and the force sensor showed to provide reliable information about catheter advancing and indication about catheter-endocardium contact.
\end{abstract}

\section{Introduction}

The insertion and manoeuvring of electrophysiology (EP) catheters in the human body for diagnostic and/or therapeutic purposes is typically carried out manually by an operator who introduces catheters with unidirectional or bidirectional deflection capability into the cardiovascular system with single or combined movements of advance/withdrawal, rotation and steering of the tip. While standing at the patient's side, the operator navigates the catheter to the desired position under imaging systems aided by X-ray fluoroscopy [1], while relying on his own skill and sensitivity to detect any impediments to catheter advancing.

The use of robotic systems in cardiac interventional procedures is growing. Recent applications dealing with remote manipulation of EP catheters report the use of a magnetically-guided catheter navigation system (NIOBE II System, Stereotaxis Inc., St.Louis, MO, USA) [2-4] and of a robotically controlled catheter system (Sensei, Hansen Medical Inc., Mountain View, CA, USA) [5-7]. However, these systems require dedicated catheters and devices.

This study aims at evaluating the feasibility and safety of a novel Telerobotic System (TS) to remotely manipulate standard steerable EP catheters, through a series of animal trials.

\section{Methods}

A TS was developed to remotely control standard steerable EP catheters without the need of dedicated guide catheter and/or cumbersome devices. The TS (prototype CathROB08, Tre Esse Progettazione Biomedica s.r.l., Bologna, IT) is an electromechanical device which allows the operator to remotely control the catheter movements from a X-ray shielded workstation, as previously demonstrated [8]. The TS is composed of a driving unit, an electrically powered control unit and a user interface [Fig.1]. The driving unit mainly consists of controllable actuators for transmitting the following to the catheter: 1) a longitudinal movement of advance/withdrawal; 2) a clockwise/counter-clockwise rotating movement about the catheter longitudinal axis; 3 ) a steering movement of the catheter tip. The catheter is mounted on the driving unit, which is fixed to the operating bed by means of an articulated arm. The control unit mainly comprises power supply circuitry and a programmable controller (CompactRIO, National Instruments, Austin, TX), which was suitably programmed to control the catheter movement combinations and the whole robotic system. The operator can remotely control the catheter movements from a Xray shielded workstation by using a joystick connected to the control unit. A graphical interface implemented in LabVIEW (National Instruments, Austin, TX) allows the user to easily control the operating parameters during navigation [Fig.2a].

The driving unit of the TS was equipped with a force sensor (Micro Switch Force Sensor, FS Series, Honeywell, IL, USA) to measure the resistance encountered by the catheter while advancing. This component was introduced to overcome the lack of tactile sensation and ultimately prevent cardiac tissue damage during the robotically-guided procedure. 


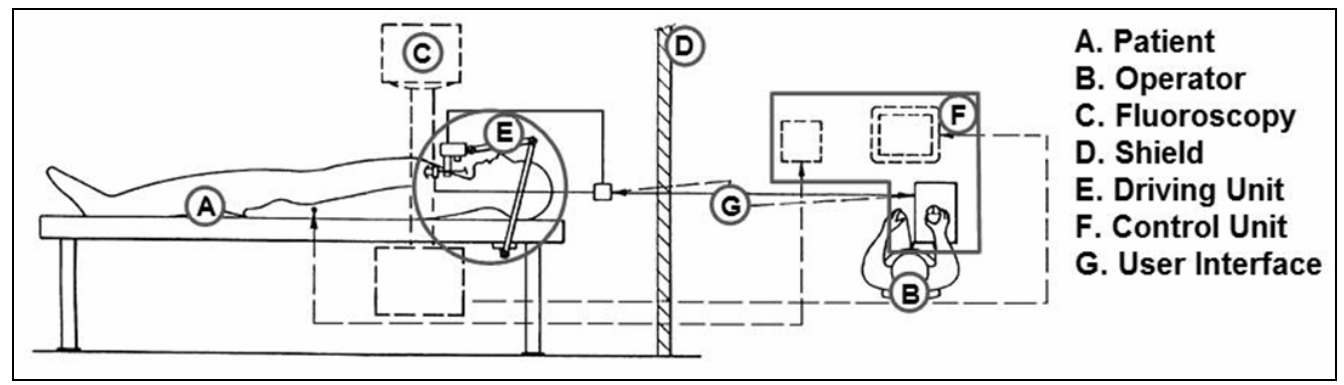

Figure 1. Schematic representation of the TS installed in the electrophysiology lab.
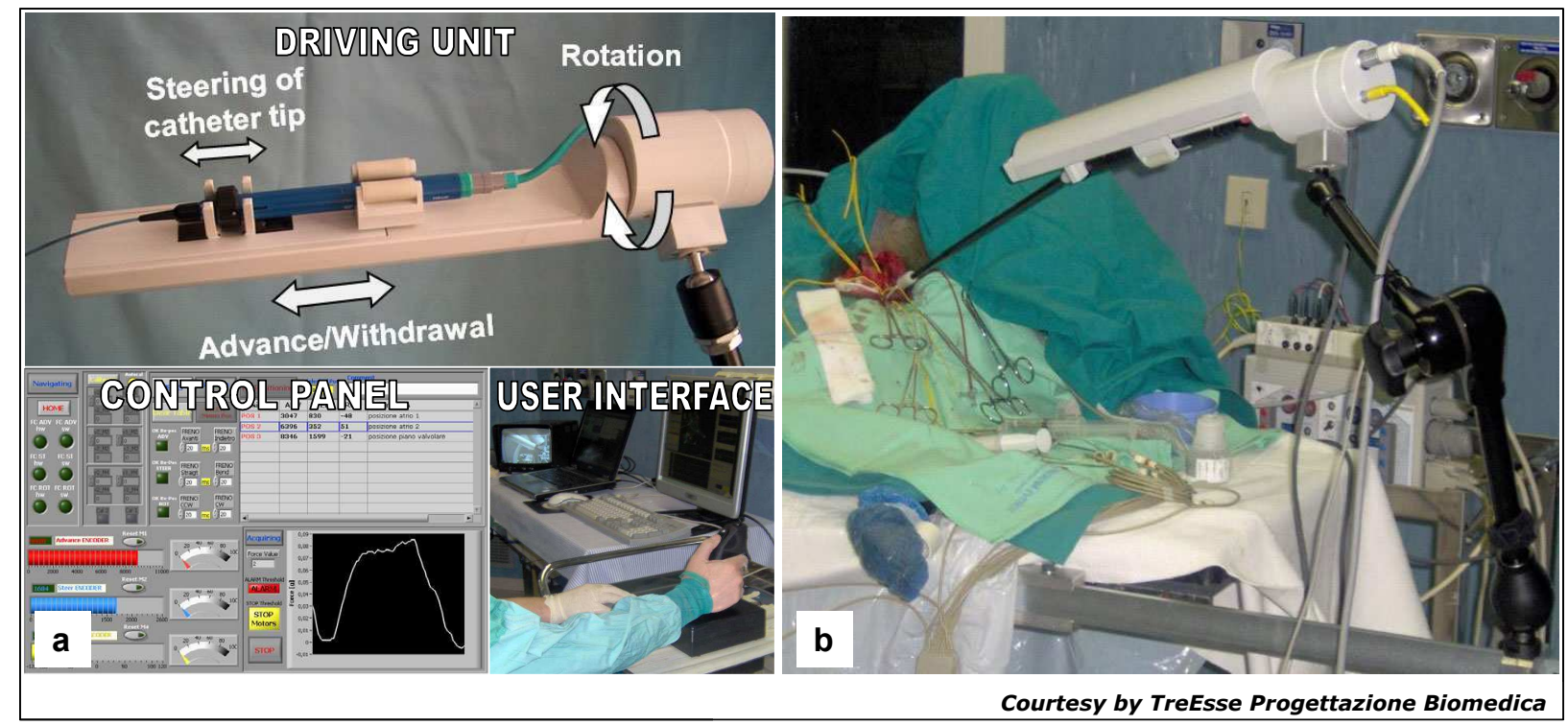

Figure 2. a) Main components of the TS: the driving unit; the user interface; the graphical control panel. b) Animal trial with the TS.

In order to evaluate the feasibility and safety of navigating EP catheter by means of the TS, three acute studies in sheep $(55 \pm 2 \mathrm{Kg})$ were performed following a standardized protocol approved by the institutional animal care and use committee [Fig.2b]. A standard EP catheter (RF Enhancr, Medtronic, Minneapolis, MN, USA) was firstly manually advanced to the Right Atrium (RA) by the operator at bedside, then remotely navigated by the TS to two selected catheter-endocardium contact targeted RA sites ( $s R A$, right atrial septum; $h R A$, high right atrium), starting from a reference catheterendocardium non-contact position close to the tricuspid annulus $(t a)$. Through an algorithm implemented in the control unit, automatic catheter repositioning to these two explored and recorded targets $(s R A 1-h R A 1)$ was performed, each time re-starting from the reference non- contact catheter position (ta1, ta2). For each advancing/withdrawing movement of the catheter, the force signal (F), measured by the force sensor on the TS, was continuously monitored and acquired. Impedance (Z), which is commonly used to evaluate catheterendocardium contact, was also measured from the EP catheter electrodes, using an impedance transducer (Model 2364, Medtronic, Minneapolis, MN, USA). The analyzed $\mathrm{F}$ and $\mathrm{Z}$ values referred to steady-state conditions and were obtained by averaging 20 samples of the acquired signals. For each sequential positioning of the catheter (ta-sRA-hRA-tal-sRA1-hRA1-ta2) percentages of variation of the force signal $(\triangle \mathrm{F})$ relative to the reference $t a$ position were measured and evaluated against percentages of variation of the impedance signal $(\Delta \mathrm{Z})$, relative to the same reference position. 


\section{Results}

Remote catheter navigation under fluoroscopic guidance by means of the TS was achieved for all predefined targets (ta-sRA-hRA-ta1-sRA1-hRA1-ta2). An example of realtime $\mathrm{F}$ and $\mathrm{Z}$ waveform charts acquired during navigation and automatic catheter repositioning is reported in Figure 3. The force signal (see Table 1 for the representative averaged $\mathrm{F}$ values) showed to provide highly reliable information about catheter advancing inside heart chambers. Trends in $F(p<0.005)$ were observed between the targeted contact catheter positions $(s R A, h R A)$ and the non-contact reference ta position (percentages of variation $\Delta \mathrm{F}=216 \%, 222 \%, 150 \%$, for sheep 1, 2, 3, respectively) [Fig.4]. Furthermore, Z measurements showed variations between contact and non-contact catheter positions, but these were less sensitive and repeatable than force measurements (percentages of variation $\Delta \mathrm{Z}=4 \% \mathrm{p}=0.008, \Delta \mathrm{Z}=103 \%$ $\mathrm{p}<0.005, \quad \Delta \mathrm{Z}=75 \% \quad \mathrm{p}<0.005$ for sheep $1, \quad 2, \quad 3$, respectively).

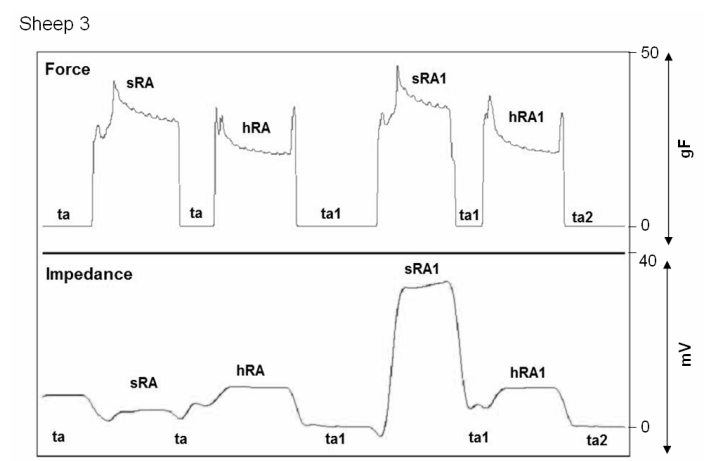

ta = tricuspid annulus; $\mathrm{sRA}=$ rigth atrial septum; $\mathrm{hRA}=$ high right atrium; ta1 = "ta" after first positioning; SRA1 = repositioning to SRA; hRA1= repositioning to hRA; ta2 = "ta" after reposititioning

Figure 3. Realtime $\mathrm{F}$ and $\mathrm{Z}$ waveform charts acquired during navigation and automatic catheter repositioning, reported for sheep 3.

\begin{tabular}{|l|c|c|c|}
\hline & SHEEP 1 & SHEEP 2 & SHEEP 3 \\
\hline Site & Force $(\mathrm{gF})$ & Force $(\mathrm{gF})$ & Force $(\mathrm{gF})$ \\
\hline ta & 0.0 & 0.0 & 0.0 \\
\hline sRA & 40.6 & 40.4 & 39.7 \\
\hline hRA & 63.4 & 65.0 & 27.0 \\
\hline ta1 & 0.0 & 0.1 & 0.0 \\
\hline sRA1 & 40.0 & 39.9 & 43.9 \\
\hline hRA1 & 61.3 & 65.0 & 28.5 \\
\hline ta2 & 0.0 & 0.0 & 0.0 \\
\hline
\end{tabular}

Table1. Force values (gF) measured during navigation and automatic catheter repositioning to the targeted sites (values averaged over 20 samples for each site).

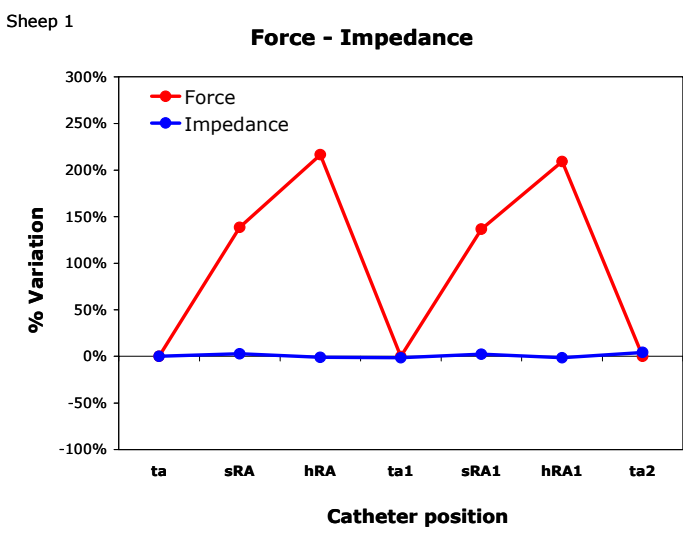

ta = tricuspid annulus; $\mathbf{S R A}=$ rigth atrial septum; $\mathbf{h R A}=$ high right atrium; ta1 = "ta" after reposititioning.

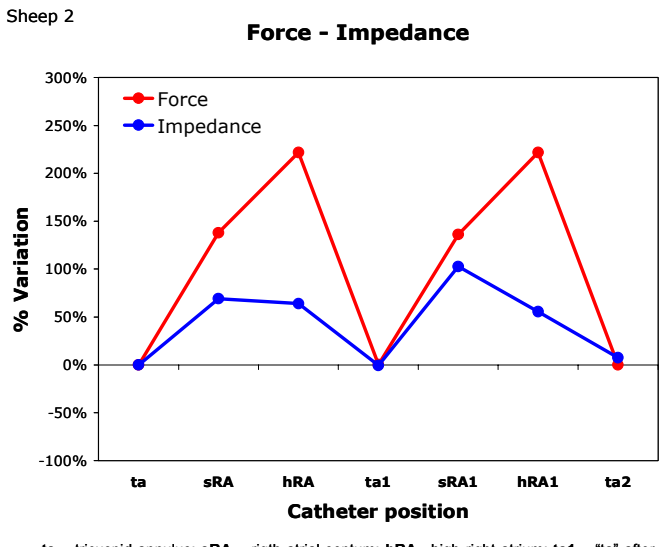

ta = tricuspid annulus; $\mathbf{S R A}=$ rigth atrial septum; $\mathbf{h R A}=$ high right atrium; ta1 $=$ "ta" after
first positioning; sRA1 = repositioning to SRA; $\mathrm{hRA}=$ repositioning to hRA; ta2 = "ta" after reposititionin

Sheep 3
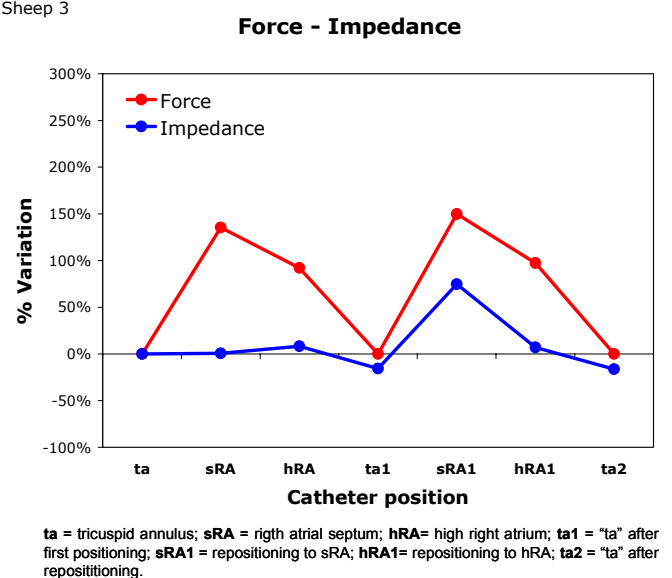

Figure 4. Percentages of $\mathrm{F}$ and $\mathrm{Z}$ variation for sheep 1, 2, 3 (top, middle, bottom) during catheter navigation (targets sRA, hRA) and automatic catheter reposition to the same targets (sRA1, hRA1) by means of the TS. 


\section{Discussion and conclusions}

The TS, equipped with an on-board force sensor, proved to be a promising tool for safe remote navigation of standard steerable EP catheters. It may help to prevent the risk of cardiac tissue damage and distinguish between contact and non-contact catheter-endocardium positions. In order to provide a feedback mechanism while robotically controlling the catheter, we used a force sensor mounted on the driving unit of the TS. The major limitation of this approach is that force measurements are taken at the proximal end of the catheter rather than at the distal catheter tip, taking into account also all unwanted frictional forces encountered by the catheter shaft while advancing. This unwanted component should be subtracted from the acquired force signal to obtain information only related to catheter-endocardium contact. When the catheter orientation is perpendicular to the cardiac surface, the point load from the distal catheter tip is entirely and directly transmitted to the proximal force sensor, while when the catheter is deflected the point load drops and the force transmitted to the sensor decreases. During cardiac ablation, catheter orientation is not necessarily perpendicular to the cardiac surface: in some cases it is oblique or even parallel to the surface, thus the contact force recorded with the force sensor is lower than in the case of perpendicular orientation. However, if the catheter touches the cardiac surface obliquely the risk of perforation drastically decreases and the force sensor coherently records a lower signal, keeping well below a safety threshold value. In this study we evaluated the use of the TS only for catheter guidance in the RA. However, this technology should not be intended restricted to RA, as the catheter could be driven by the same TS also in right ventricle and in the left cardiac chambers, after performing "transeptal catheterization". Future experiments will include a wide range of contact force measurements (both in atria and ventricles) and multiple repositioning to the same pre-explored and recorded endocardial target. Also clinical trials are going to be planned to confirm the feasibility and safety of navigating EP catheter by this novel TS.

\section{Acknowledgements}

The authors thank D. Golinelli, M. Panfili and F. Bortolami for their valuable input and technical support during this study. This work was supported by TreEsse Progettazione Biomedica, which provided the TS prototype for the animal trials.

\section{References}

[1] Delichas $M$ et al.. Radiation exposure to cardiologists performing interventional cardiology procedures. Eur J Radiol. 2003; 48(3):268-73.

[2] Faddis MN et al.. Magnetic guidance system for cardiac electrophysiology: a prospective trial of safety and efficacy in humans. J Am Coll Cardiol 2003; 42(11):1952-8.

[3] Faddis MN et al.. Novel, magnetically guided catheter for endocardial mapping and radiofrequency catheter ablation. Circulation 2002, 106(23):2980-5.

[4] Pappone $\mathrm{C}$ et al.. Robotic magnetic navigation for atrial fibrillation ablation. J Am Coll Cardiol 2006; 47(7):1390400.

[5] Al-Ahmad A et al.. Early experience with a computerized robotically controlled catheter system. J Interv Card Electrophysiol 2005; 12(3):199-202.

[6] Saliba W et al.. Novel robotic catheter remote control system: feasibility and safety of transseptal puncture and endocardial catheter navigation. J Cardiovasc Electrophysiol 2006; 17(10):1102-5.

[7] Kanagaratnam $\mathrm{P}$ et al.. Experience of robotic catheter ablation in humans using a novel remotely steerable catheter sheath. J Interv Card Electrophysiol., 2008; 21(1):19-26.

[8] Cercenelli L et al.. Initial experience with a telerobotic system to remotely navigate and automatically re-position standard steerable EP catheters. Asaio J, 2007; 53(5): 523529.

Address for correspondence

Emanuela Marcelli

Università di Bologna

Dipartimento Clinico di Scienze Radiologiche e

Istocitopatologiche - Sezione Tecnologie Biomediche

Policlinico S. Orsola Malpighi

Via Massarenti, 9 (Palazzina 17)

40138 Bologna, Italy

emanuela.marcelli@unibo.it 